\title{
Predictors, rates, and trends of opioid use disorder among patients hospitalized with chronic pancreatitis
}

\author{
Adeyinka Charles Adejumo a,b,* Olalekan Akanbic,* Quazim Alayo ${ }^{d}$, Victor Ejigahe, \\ Nnaemeka Egbuna Onyeakusif, Ogorchukwu Faith Omede ${ }^{\mathrm{a}, \mathrm{b}}$, Lydie Pani ${ }^{\mathrm{a}, \mathrm{b}}$, Oluwatosin Omole
}

North Shore Medical Center, Salem, Massachusetts; Tufts University Medical School, Boston, Massachusetts; University of Kentucky College of Medicine, Lexington Kentucky; St. Luke's Hospital, Chesterfield, Missouri; University of Massachusetts Lowell, Lowell, Massachusetts; Case Western - MetroHealth campus, Cleveland, Ohio; University Health System, San Antonio, Texas, USA

Abstract

Background Patients with chronic pancreatitis (CP) suffer from pain and receive increased opioid prescriptions with a high risk of opioid use disorder (OUD). We studied the predictors, trends and outcomes of OUD among patients hospitalized with CP.

Methods Records with CP (with/without OUD) were extracted from the Nationwide Inpatient Sample (NIS) 2012-2014, and the association of OUD with the burden of CP was calculated. We then charted the trends of OUD and its interaction with concomitant CP from NIS 2007-2014 (SAS 9.4).

Results In the period 2012-2014, 4349 (4.99\%) of the 87,068 CP patients had concomitant OUD, with higher risk among patients who were young, females, white vs. Hispanics, and individuals with chronic back pain, arthritis, non-opioid substance use, mental health disorders, and those hospitalized in urban centers. OUD was associated with a longer hospital stay (6.9 vs. 6.5 days, $\mathrm{P}=0.0015)$ but no significant difference in charges $(\$ 47,151$ vs. $\$ 49,017, \mathrm{P}=0.0598)$ or mortality ( $1.64 \%$ vs. $0.74 \%, P=0.0506$ ). From $2007-2014$, the average yearly rate of OUD was 174 cases per 10,000 hospitalizations (174/10,000), almost 3 times higher among CP vs. non-CP (479/10,000 vs. $173 / 10,000, \mathrm{P}<0.001)$, and it increased from 2007 to $2014(135 / 10,000$ to $216 / 10,000, \mathrm{P}<0.001)$. The yearly increase was 2.7 times higher among patients with CP vs. non-CP (29.9/10,000 vs. 11.3/10,000 hospitalizations/year, $\mathrm{P}<0.001)$.

Conclusions $\mathrm{CP}$ is associated with higher rates and trends of OUD. Patients with CP at high risk of OUD may benefit from alternate analgesic regimens or surveillance for OUD when they are prescribed opioids.

Keywords Length of stay, pain, mental health, charges, mortality

Ann Gastroenterol 2021; 35 (2): 1-11

Department of ${ }^{a}$ Medicine, North Shore Medical Center, Salem, Massachusetts (Adeyinka Charles Adejumo, Ogorchukwu Faith

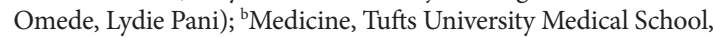
Boston, Massachusetts (Adeyinka Charles Adejumo, Ogorchukwu Faith Omede, Lydie Pani); ' ${ }^{M e d i c i n e, ~ U n i v e r s i t y ~ o f ~ K e n t u c k y ~ C o l l e g e ~}$ of Medicine, Lexington Kentucky (Olalekan Akanbi); ${ }^{\mathrm{d}}$ Medicine, St. Luke’s Hospital, Chesterfield, Missouri (Quazim Alayo); ${ }^{e}$ Pharmaceutical Sciences, University of Massachusetts Lowell, Lowell, Massachusetts (Victor Ejigah); ${ }^{\mathrm{f} A n e s t h e s i o l o g y, ~ C a s e ~ W e s t e r n ~-~ M e t r o H e a l t h ~ c a m p u s, ~}$ Cleveland, Ohio (Nnaemeka Egbuna Onyeakusi); ${ }^{\mathrm{F} F a m i l y ~ M e d i c i n e, ~}$ University Health System, San Antonio, Texas (Oluwatosin Omole), USA

Conflict of Interest: None

Correspondence to: Adeyinka Charles Adejumo, MD, MS, Division of Gastroenterology, Hepatology and Nutrition, Department of Medicine, University of Pittsburgh School of Medicine, Pittsburgh, PA, USA, e-mail: adeyinka_adejumo@yahoo.com

Received 18 August 2019; accepted 23 September 2020; published online 16 January 2021

DOI: https://doi.org/10.20524/aog.2021.0579

\section{Introduction}

Opioids are commonly prescribed for the management of chronic pain in the United States and there has been a surge in prescriptions over the last 2 decades [1-3]. However, as the prescription rates have increased, the potential for abuse and ensuing untoward effects of these medications has assumed significant public health significance [4]. According to the Centers for Disease Control and Prevention (CDC), 11.5 million Americans in 2016 self-reported misuse of prescription opioids, while 2.1 million had opioid use disorder (OUD), as defined in the Diagnostic and Statistical Manual of Mental Disorders, $5^{\text {th }}$ Edition (DSM-5) as a problematic pattern of opioid use leading to clinically significant impairment or distress [5]. Furthermore, chronic opioid therapy is associated with higher mortality [6,7], amongst many other opioid-related adverse events that result in higher numbers of emergency room visits, prolonged hospital stays, increased costs, and healthcare 
resource utilization [3,8-12], without necessarily providing lasting pain relief, or any improvement in quality of life or functional capacity [13]. The CDC has published prescription guidelines, while states have prescription monitoring programs in efforts designed to mitigate the scourge of inappropriate prescription and OUD $[14,15]$.

Chronic pancreatitis (CP) is a complex inflammatory disease characterized by lasting, progressive scarring and irreversible damage to the pancreas, resulting in loss of function [16,17]. Consequently, it is an incurable condition with treatment focused on the alleviation of pain and management of complications relating to malabsorption and insulin dependence $[16,18,19]$. Patients with CP commonly present across various healthcare settings with recurrent abdominal pain due to pancreatic inflammation, hyperstimulation and neuropathy, contributing to a considerably decreased quality of life. As a result, healthcare providers are often faced with the challenging task of assessing and managing this debilitating pain $[18,20]$. Despite best efforts, including pharmacologic, endoscopic and surgical interventions, most patients remain symptomatic and suffer from complications [21]. Narcotics are frequently utilized as a central part of a stepwise approach to providing analgesia, but they are unfortunately strongly associated with a potential for side effects and increased risk of tolerance and dependency [18,20,21]. Nusrat et al identified opioid use among 51\% of CP patients [22].

Despite the common utilization of opioids and the potential for misuse among patients with CP, the burden and risk factors for OUD among this patient group have not been evaluated in a large population study. Barth et al found that depression, quality of life, pain intensity, and alcohol use were associated with opioid misuse among patients with chronic non-alcoholic pancreatitis [23]. However, their study was limited to a subset of patients with CP in a single tertiary center. We therefore sought to estimate the national prevalence and trends of OUD among patients hospitalized with $\mathrm{CP}$, using a nationwide analysis, and to identify clinical and demographic predictors of OUD in this patient population.

\section{Patients and methods}

\section{Data source and study design}

This study was a retrospective cross-sectional study of all hospital admissions with a discharge diagnosis of CP between 2012 and 2014. Data were extracted from the National Inpatient Database (NIS), maintained by the Agency for Healthcare Research and Quality (AHRQ) as a core component of the Healthcare Cost and Utilization Project (HCUP). The AHRQ also performs validation and quality control of the database [24]. As the largest publicly available all-payer inpatient healthcare database in the USA, the NIS provides national estimates of more than 7 million discharges per annum [24]. It also provides valuable data on patient demographics, 30 primary and secondary diagnoses, 15 inpatient procedures, hospital characteristics, insurance status, comorbidity measures, hospitalization charges, and length of stay, while protecting identifying patient information [24]. The diagnoses and procedures were encoded with the International Classification of Diseases, Ninth Revision, Clinical Modification (ICD-9$\mathrm{CM}$ ). The use of the NIS is exempt from an Institutional Review Board as there is no identifying patient information $[25,26]$.

\section{Study population}

All adult (age $\geq 18$ years) hospitalizations for CP from NIS 2012-2014 were used for the cross-sectional study, while all adult (age $\geq 18$ years) hospitalizations from NIS 2007-2014 were used for the trend analysis (Fig. 1). Patients with comorbid CP were identified by the ICD-9-CM code 577.1, while those with comorbid OUD were identified by the ICD-9-CM codes 304.0, 304.7 and 305.5 [27-29].

\section{Variables}

Patients' characteristics were identified from the NIS: demographics, hospitalization, hospital level, and comorbid diseases. Demographic factors included were age, sex (male and female), race/ethnicity (Whites, Blacks, Hispanics, and

All records in the 2012-2014 HCUP-NIS Data set $(21,488,293)$

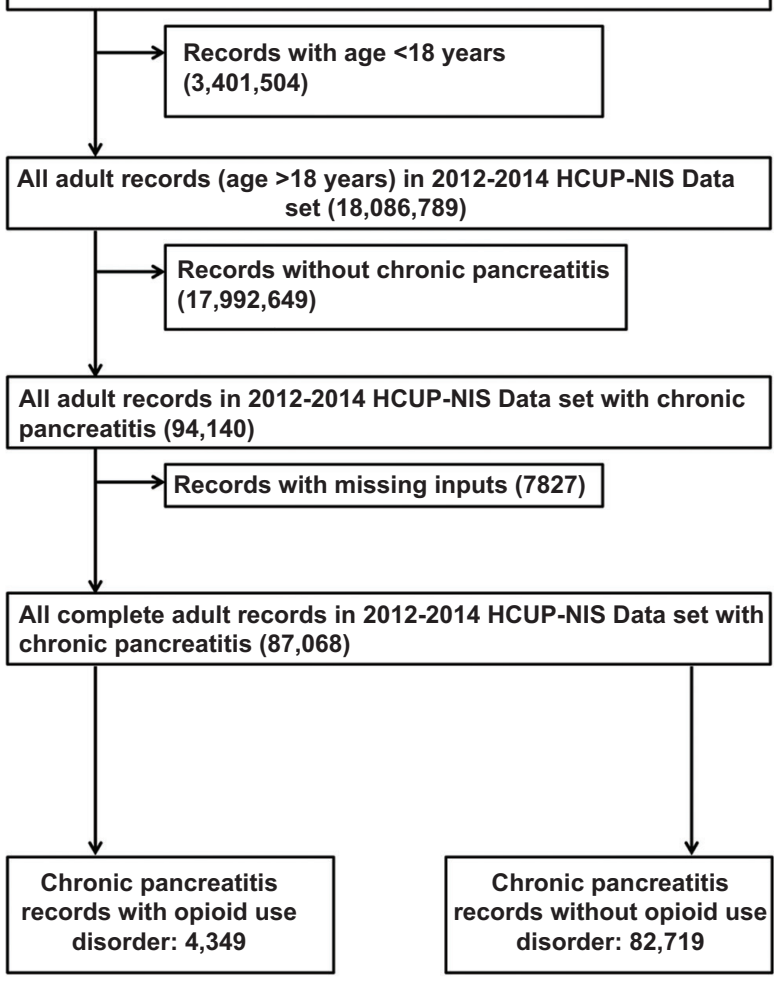

Figure 1 Selection flowchart for hospitalizations with chronic pancreatitis in the United States, NIS 2007-2014

NIS, National Inpatient Database (NIS); HCUP, Healthcare Cost and Utilization Project 
other races), health insurance (Medicare, Medicaid, private, self-pay \& others), income status (categorized into 4 according to the average household income of the zip-code where the patient lives), and geographic region (Northeast, Midwest, South and West). Hospitalization factors include whether the admission was during the weekend (no and yes), was a transfer from another facility (not a transfer, transfer from another acute care hospital, or transfer from a secondary/ rehabilitation center), was an elective admission (no and yes), and in which season (autumn, winter, spring and summer). Hospital characteristics included the teaching status (rural non-teaching, urban non-teaching, and urban teaching), number of beds and hospital financial control. Based on the American Hospital Association annual survey of hospitals, the hospitals were categorized into 3 according to the number of acute care beds set up, hospital teaching status, and geographic region [30]. Roughly, small hospitals ranged from 1-249 beds, medium from 50-449 and large with 100+ beds, depending on the geographic region and teaching status. Similarly, hospital financial control was segregated into 3: public hospitals were categorized as government, voluntary hospitals as private non-profit, and proprietary hospitals as private profit [31]. Comorbid diseases were identified with ICD-9-CM codes (Supplementary Table 1) and included mental health disorders, chronic back pain, non-opioid substance abuse, chronic arthritis pain, and chronic headache. Possible etiologies of $\mathrm{CP}$ were identified and categorized int 3 groups: alcohol use, idiopathic, and others (biliary stones, family history of pancreatitis, autoimmune diseases, pancreatic cancer, hypercalcemia, hypertriglyceridemia, and cystic fibrosis). We also identified different comorbid diseases across all the organ systems and summarized them based on the Charlson-Deyo index [32]. Many of the comorbidities in this study have been used in many studies in the NIS [33-36].

The outcomes of the study were the prevalence of OUD, trends of OUD, and length of stay (LOS) during hospitalization.

\section{Statistical analysis}

The NIS data were extracted and analyzed using the Statistical Analysis System (SAS V.9.4, SAS Institute Inc, Cary, NC), and accounted for the complex multilevel survey methodology in the NIS $[37,38]$ by eliminating the effect of the clustering of patients within the same hospital and hospitals within similar strata. Analyses incorporated recommendations from AHRQ [37], accounting for the complex survey methodology [38]. Numeric variables were summarized with mean and standard deviation and compared with Student's $t$-test, while categorical variables were summarized with percentages and compared using the Rao-Scott Chi-square test. Predictors of OUD among CP patients were identified with a multivariate survey logistic regression model with OUD as the outcome, and patients and hospital characteristics as the explanatory variables. Among CP patients, the association of OUD and mortality was calculated using multivariate generalized models, adjusting for demographic, comorbid, and hospital level covariates. Similarly, multivariate models were used to calculate the association between OUD and LOS (negative binomial model) and charges (gamma models). Among all adult hospitalizations from 2007-2014, the yearly frequency of OUD was calculated and charted. The trend line was divided into 2 based on the presence of $\mathrm{CP}$, then the interaction of CP with the trends of OUD was calculated using generalized linear models (Poisson distribution).

\section{Results}

\section{Baseline characteristics}

The baseline characteristics of the study population are presented in Table 1. In the years 2012-2014, we identified 87,068 patients with a primary discharge diagnosis of CP and stratified them by the presence or absence of concomitant OUD (Fig. 1). Of these patients, 4349 (4.99\%) also had OUD. CP patients with or without OUD were predominantly male, White and were predominantly in the lowest quartile of income. CP patients with OUD were younger (mean age $46.9 \pm 11.73$ vs. $52.6 \pm 14.72$ years), and had a lower proportion of Medicareand private-insured, but a higher proportion of Medicaidinsured patients compared to those without OUD. The West and Northeast regions had a higher proportion of patients with OUD. Compared with those without OUD, CP patients with opioid abuse were more likely to be admitted over the weekend and to be admitted non-electively. Urban, privately controlled non-profit hospitals had the highest proportions of patients with CP and OUD.

In addition, patients with CP and OUD were more likely to engage in non-opioid substance abuse (alcohol and nonopioid drugs) and included a higher proportion of patients with mental disorders. Although this group of patients had more pain-associated conditions, such as chronic back pain and headache, they had a smaller comorbidity burden in comparison to CP patients without OUD. There was no significant difference between the groups as regards hospital size or transfer admission status. Regarding the etiology and risk factors of $\mathrm{CP}$, patients with alcohol use disorder were more likely to have OUD, while other risk factors for CP (idiopathic, and others) were higher among those without OUD (Table 1). All substance use disorders (alcohol, tobacco and other substance) were more predominant among subjects with OUD.

\section{Predictors of opioid use disorder}

Table 2 shows the demographic and clinical factors significantly associated with OUD in patients hospitalized with $\mathrm{CP}$. On multivariate analysis, age, sex and race were independent predictors of OUD. Increasing age was associated with $21 \%$ (adjusted odds ratio [aOR] $0.79,95 \%$ confidence interval [CI] 0.77-0.80) reduced odds of OUD while females had $6 \%$ greater odds compared to males. While Hispanic 
4 A. C. Adejumo et al

Table 1 Characteristics of hospitalizations with chronic pancreatitis by opioid use disorder, NIS 2012-2014

\begin{tabular}{|c|c|c|c|}
\hline Characteristics & $\begin{array}{c}\text { No-opioid } \\
82,719(\sim 413,595)\end{array}$ & $\begin{array}{c}\text { Opioid } \\
4349(\sim 21,745)\end{array}$ & P-value \\
\hline Age, mean (SD), y & $52.63(14.72)$ & $46.90(11.73)$ & $<0.001$ \\
\hline Age, categorized, y & & & $<0.001$ \\
\hline $18-45$ & 18.84 & 27.41 & \\
\hline $45-65$ & 51.71 & 59.44 & \\
\hline$>65$ & 29.44 & 13.15 & \\
\hline Sex, \% & & & 0.3160 \\
\hline Male & 55.04 & 54.20 & \\
\hline Female & 44.96 & 45.80 & \\
\hline Race, \% & & & 0.0157 \\
\hline White & 64.90 & 67.26 & \\
\hline Black & 23.04 & 21.94 & \\
\hline Hispanic & 7.79 & 7.40 & \\
\hline Other & 4.27 & 3.40 & \\
\hline Insurance, \% & & & $<0.001$ \\
\hline Medicare & 39.43 & 33.85 & \\
\hline Medicaid & 24.26 & 35.25 & \\
\hline Private & 21.99 & 17.52 & \\
\hline Self-pay \& others ${ }^{*}$ & 14.32 & 13.38 & \\
\hline Income, $\%$ & & & 0.0799 \\
\hline Lowest quartile & 36.34 & 34.90 & \\
\hline Second quartile & 26.39 & 26.17 & \\
\hline Third quartile & 20.93 & 21.06 & \\
\hline Highest quartile & 16.34 & 17.87 & \\
\hline Region, \% & & & $<0.001$ \\
\hline Northeast & 18.34 & 23.66 & \\
\hline Midwest & 22.79 & 20.44 & \\
\hline South & 42.09 & 32.95 & \\
\hline West & 16.78 & 22.95 & \\
\hline Admission at weekend, $\%$ & & & 0.0031 \\
\hline No & 76.47 & 74.52 & \\
\hline Yes & 23.53 & 25.48 & \\
\hline Season, \% & & & 0.7002 \\
\hline Autumn & 25.01 & 25.78 & \\
\hline Winter & 24.90 & 24.47 & \\
\hline Spring & 25.89 & 25.57 & \\
\hline Summer & 24.21 & 24.19 & \\
\hline Transfer admission, \% & & & 0.2016 \\
\hline No & 92.88 & 92.97 & \\
\hline From hospital & 5.15 & 5.44 & \\
\hline From secondary health facility & 1.97 & 1.60 & \\
\hline
\end{tabular}

(Contd...) 
Table 1 (Continued)

\begin{tabular}{|c|c|c|c|}
\hline Characteristics & $\begin{array}{c}\text { No-opioid } \\
82,719(\sim 413,595)\end{array}$ & $\begin{array}{c}\text { Opioid } \\
4349(\sim 21,745)\end{array}$ & P-value \\
\hline Elective admission, \% & & & $<0.001$ \\
\hline No & 90.95 & 93.21 & \\
\hline Yes & 9.05 & 6.79 & \\
\hline Hospital teaching status, $\%$ & & & $<0.001$ \\
\hline Rural & 10.04 & 6.58 & \\
\hline Urban non-teaching & 31.41 & 32.79 & \\
\hline Urban teaching & 58.54 & 60.63 & \\
\hline Hospital size, $\%$ & & & 0.1973 \\
\hline Small & 14.83 & 14.14 & \\
\hline Medium & 27.63 & 26.58 & \\
\hline Large & 57.54 & 59.28 & \\
\hline Hospital control, \% & & & 0.0037 \\
\hline Government & 12.88 & 11.45 & \\
\hline Private, non-profit & 73.35 & 76.50 & \\
\hline Private, profit & 13.77 & 12.05 & \\
\hline Mental heath disorders, $\%$ & 28.11 & 46.29 & $<0.001$ \\
\hline Chronic back pain, $\%$ & 9.05 & 13.06 & $<0.001$ \\
\hline \multicolumn{4}{|l|}{ Etiology of chronic pancreatitis, \% } \\
\hline Alcoholic & 46.56 & 60.57 & $<0.001$ \\
\hline Idiopathic & 45.17 & 34.42 & $<0.001$ \\
\hline Other causes/risk factors & 12.87 & 9.70 & $<0.001$ \\
\hline Biliary stones & 4.92 & 2.71 & $<0.001$ \\
\hline Family history of pancreatitis & 0.11 & 0.07 & 0.4567 \\
\hline Autoimmune & 1.52 & 1.40 & 0.5598 \\
\hline Pancreatic cancer & 1.75 & 0.97 & $<0.001$ \\
\hline Hypercalcemia & 0.76 & 0.85 & 0.4952 \\
\hline Hypertriglyceridemia & 3.79 & 2.94 & 0.0045 \\
\hline Cystic fibrosis & 0.50 & 0.90 & $<0.001$ \\
\hline Tobacco use, $\%$ & 46.78 & 55.51 & $<0.001$ \\
\hline Other substance use (not alcohol, tobacco nor opioid), \% & 9.52 & 36.68 & $<0.001$ \\
\hline Charlson-Deyo comorbidity index, \% & & & $<0.001$ \\
\hline Deyo: 0 & 32.30 & 37.96 & \\
\hline Deyo: $1-3$ & 48.83 & 47.30 & \\
\hline Deyo: $>3$ & 18.87 & 14.74 & \\
\hline
\end{tabular}

*Self-pay \& others: Individuals without a health insurance or paying health fees out of pocket

$S D$, standard deviation, NIS, National Inpatient Database

ethnicity was associated with lower odds of OUD (aOR 0.84, 95\%CI 0.77-0.93), Blacks and other ethnic groups were similar to Whites. Relative to individuals with private insurance, patients insured by Medicare or Medicaid had significantly higher odds of OUD, while those categorized as "Others" (selfpay and uninsured) showed no difference. Compared to the Northeast, the Midwest and South were both associated with
32-35\% lower odds of opioid abuse amongst hospitalized CP patients, while the West had no difference. Urban teaching and non-teaching hospitals were significantly associated with OUD compared to rural. Individual comorbid conditions, such as mental health disorders, back pain, arthritis and headaches were significant predictors of OUD. Likewise, alcohol use and use of other substances (not alcohol, tobacco or opioid) 
Table 2 Predictors of opioid use disorder among hospitalizations with chronic pancreatitis, NIS 2012-2014

\begin{tabular}{|c|c|c|}
\hline Predictors & Adjusted odds ratio $(95 \% \mathrm{CI})$ & P-value \\
\hline Age, per 10-year increase & $0.79(0.77-0.80)$ & $<0.001$ \\
\hline Sex: female vs. male & $1.06(1.01-1.11)$ & 0.0163 \\
\hline Race & & 0.0013 \\
\hline Black vs. White & $0.95(0.89-1.01)$ & \\
\hline Hispanics vs. Whites & $0.84(0.77-0.93)$ & \\
\hline Others vs. Whites & $0.90(0.79-1.01)$ & \\
\hline Health insurance & & $<0.001$ \\
\hline Medicare vs. private & $1.29(1.20-1.38)$ & \\
\hline Medicaid vs. private & $1.36(1.27-1.45)$ & \\
\hline Self-pay \& others ${ }^{\star}$ vs. private & $0.97(0.89-1.05)$ & \\
\hline Income status & & 0.0205 \\
\hline Lowest vs. highest quartile & $1.01(0.95-1.07)$ & \\
\hline Second vs. highest quartile & $1.01(0.95-1.08)$ & \\
\hline Third vs. highest quartile & $1.11(1.03-1.19)$ & \\
\hline Weekend vs. weekday admission & $1.03(0.97-1.08)$ & 0.3412 \\
\hline Season & & 0.0872 \\
\hline Autumn vs. winter & $1.03(0.97-1.10)$ & \\
\hline Spring vs. winter & $0.97(0.91-1.04)$ & \\
\hline Summer vs. winter & $0.95(0.89-1.02)$ & \\
\hline Transfer status & & 0.1597 \\
\hline From acute care hospitals vs. none & $1.01(0.91-1.13)$ & \\
\hline From secondary care facilities vs. none & $0.82(0.67-1.01)$ & \\
\hline Non-elective vs. elective admission & $1.08(0.99-1.17)$ & 0.1007 \\
\hline Hospital region & & $<0.001$ \\
\hline Midwest vs. Northeast & $0.65(0.60-0.69)$ & \\
\hline South vs. Northeast & $0.68(0.64-0.72)$ & \\
\hline West vs. Northeast & $1.05(0.98-1.13)$ & \\
\hline Hospital teaching status & & $<0.001$ \\
\hline Urban non-teaching vs. rural & $1.37(1.25-1.51)$ & \\
\hline Urban teaching vs. rural & $1.46(1.33-1.60)$ & \\
\hline Hospital size & & 0.2244 \\
\hline Medium vs. small & $1.07(0.99-1.15)$ & \\
\hline Large vs. small & $1.06(0.99-1.13)$ & \\
\hline Hospital control & & $<0.001$ \\
\hline Private non-profit vs. government & $1.14(1.06-1.23)$ & \\
\hline Private for-profit vs. government & $0.97(0.88-1.07)$ & \\
\hline Mental health disorders & $1.66(1.58-1.74)$ & $<0.001$ \\
\hline Back pain & $1.46(1.35-1.57)$ & $<0.001$ \\
\hline Arthritis & $1.15(1.08-1.23)$ & $<0.001$ \\
\hline Headaches & $1.17(1.05-1.31)$ & 0.0057 \\
\hline Malignancy & $0.88(0.75-1.03)$ & 0.1108 \\
\hline \multicolumn{3}{|l|}{ Etiology of chronic pancreatitis } \\
\hline Alcoholic & $1.31(1.11-1.54)$ & 0.0011 \\
\hline Idiopathic & $0.96(0.81-1.12)$ & 0.5878 \\
\hline Other causes/risk factors & $0.80(0.71-0.90)$ & $<0.001$ \\
\hline Tobacco & $1.01(0.95-1.07)$ & 0.8342 \\
\hline Other substances (not alcohol, tobacco, or opioid) & $4.77(4.52-5.03)$ & $<0.001$ \\
\hline Charlson-Deyo comorbidity index & & 0.2124 \\
\hline Deyo: $1-3$ vs. 0 & $1.00(0.95-1.06)$ & \\
\hline Deyo: $>3$ vs. $1-3$ & $0.94(0.86-1.02)$ & \\
\hline
\end{tabular}

${ }^{*}$ Self-pay \& others: Individuals without a health insurance or paying health fees out of pocket

CI, confidence interval, NIS, National Inpatient Database 
were respectively associated with $31 \%(1.31,95 \%$ CI $1.11-1.54$; $\mathrm{P}=0.0011)$ and $380 \%$ (4.77, 95\%CI 4.52-5.03; $\mathrm{P}<0.001)$ increased odds of having OUD. However idiopathic causes of $\mathrm{CP}$ were not associated with OUD, and other causes of $\mathrm{CP}$ were associated with $20 \%$ lower odds of OUD (0.80 95\%CI 0.71 $0.90 ; \mathrm{P}=0.0020)$.

\section{Outcomes of CP with OUD}

Compared to patients without OUD (Table 3), those with OUD stayed longer (adjusted length of stay, 6.9 vs. 6.5 days, $\mathrm{P}=0.0015$ ), after accounting for demographic, comorbid, and hospital variables. However, there was no difference in the hospital mortality rate ( 1.64 vs. $0.74 \%, \mathrm{P}=0.0506)$ and charges between OUD and no-OUD ( $\$ 47,151$ vs. $\$ 49,017, \mathrm{P}=0.0598)$.

\section{Temporal trends of OUD}

Temporal trends of OUD were analyzed between 2007 and 2014. The rate of OUD among all patients hospitalized in the United States rose steadily from 135 to 216 cases per 10,000 hospitalizations (1.35-2.16\%), at an average rate of 11.41 cases per 10,000 hospitalizations per annum (Fig. 2A and Supplementary Table 2). When stratified by the primary diagnosis of CP (Fig. 2B), the OUD rate increased from 365 to 566 cases per 10,000 hospitalizations (3.6-5.6\%) at a gradient of 30 cases per 10,000 hospitalizations per year $(0.30 \%)$, culminating in an average frequency of $4.79 \%$. With a frequency of $1.74 \%$, OUD rates were lower in those without CP, consistently staying at below 220 cases per 10,000 hospitalizations (1.34-2.16\%) and rising by only 11.3 cases per 10,000 hospitalizations per annum (0.11\%) between 2007 and 2014. Thus, not only were individual annual rates of OUD about 2.8 times higher in patients with CP, the slope of the increase was almost 2.7 times steeper than in patients without $\mathrm{CP}$ (Fig. 2B).

\section{Discussion}

The management of chronic non-malignant pain conditions such as $\mathrm{CP}$ poses a significant challenge to clinicians, who often rely on opioids to provide relief [39]. As a result, opioid prescription has spiked significantly in the last 2 decades in the United States, directly contributing to many undesirable consequences, amongst which is OUD [2,40]. This analysis of a large national database highlights the prevalence, trends and independent predictors of OUD among patients hospitalized with CP.

In our study population, $4.99 \%$ of patients with CP had coexisting OUD: approximately 1 in 20 patients. This frequency is higher than the reported national prevalence of $0.8 \%$ [41] and the prevalence observed in specific patient subgroups, such as those with inflammatory bowel disease (IBD) (2.2\%) [42] and those undergoing cardiac surgery (0.2\%) [43]. Several significant associated factors were identified, including younger age, female sex, insurance status, hospital region, and teaching status. Other independent predictors of OUD in the study cohort were mental health disorders, back pain, arthritis, and non-opioid substance abuse. These are consistent with the findings of previous studies in other diseases [44-46]. However, a recent single-center study of patients with pancreatitis reported that the frequency of persistent opioid use was $9 \%$ among patients with recurrent acute pancreatitis, while it was absent among patients with only pancreatitis [47]. The higher frequency $(9 \%)$ in this study is probably because the patients had a closer longitudinal follow up on their prescriptions, unlike our study, which relied solely on ICD-9-CM codes. Another notable finding in this study is the steeper rise in the annual prevalence of OUD among patients with CP relative to the rest of the population. While the average annual prevalence of opioid abuse in all hospitalized patients steadily increased over the study period, the average prevalence rate in patients with $\mathrm{CP}$ was always greater and increased at approximately triple the annual rate observed in those without $\mathrm{CP}$. Thus, $\mathrm{CP}$ is a significant driver of OUD trend among hospitalized patients.

The higher frequency of OUD observed among patients with CP firmly suggests CP as a risk factor for OUD. As a prominent and recurring cause of debilitating abdominal pain, often managed with opioids, CP potentially increases the risk of opioid abuse in afflicted individuals. The independent predictors of OUD identified in this study can assist healthcare providers to screen and identify at-risk individuals. This subgroup of patients should be offered alternative modalities of pain management, such as non-opioid analgesia, nerve blocks and endoscopic therapy, coupled with pancreatic enzyme

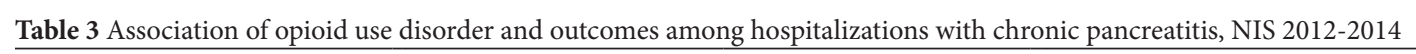

\begin{tabular}{|c|c|c|c|c|}
\hline \multirow[t]{2}{*}{ Outcome } & \multicolumn{2}{|c|}{ Opioid use } & \multicolumn{2}{|l|}{ Opioid use vs. no-use } \\
\hline & Yes & No & Adjusted Odds/Mean ratio* $(95 \% \mathrm{CI})$ & P-value \\
\hline $\begin{array}{l}\text { Length of stay, median } \\
(95 \% \mathrm{CI}) \text {, days }\end{array}$ & $6.9(6.5-7.2)$ & $6.5(6.2-6.8)$ & $1.05(1.02-1.09)$ & 0.0015 \\
\hline $\begin{array}{l}\text { Total hospital charge, median } \\
(95 \% \mathrm{CI}) \text {, \$US }\end{array}$ & $47,151(44,156-50,350)$ & $49,017(46,465-51,708)$ & $0.96(0.92-1.00)$ & 0.0598 \\
\hline In-hospital mortality, $\%$ & 1.64 & 0.74 & $0.70(0.49-1.00)$ & 0.0506 \\
\hline
\end{tabular}




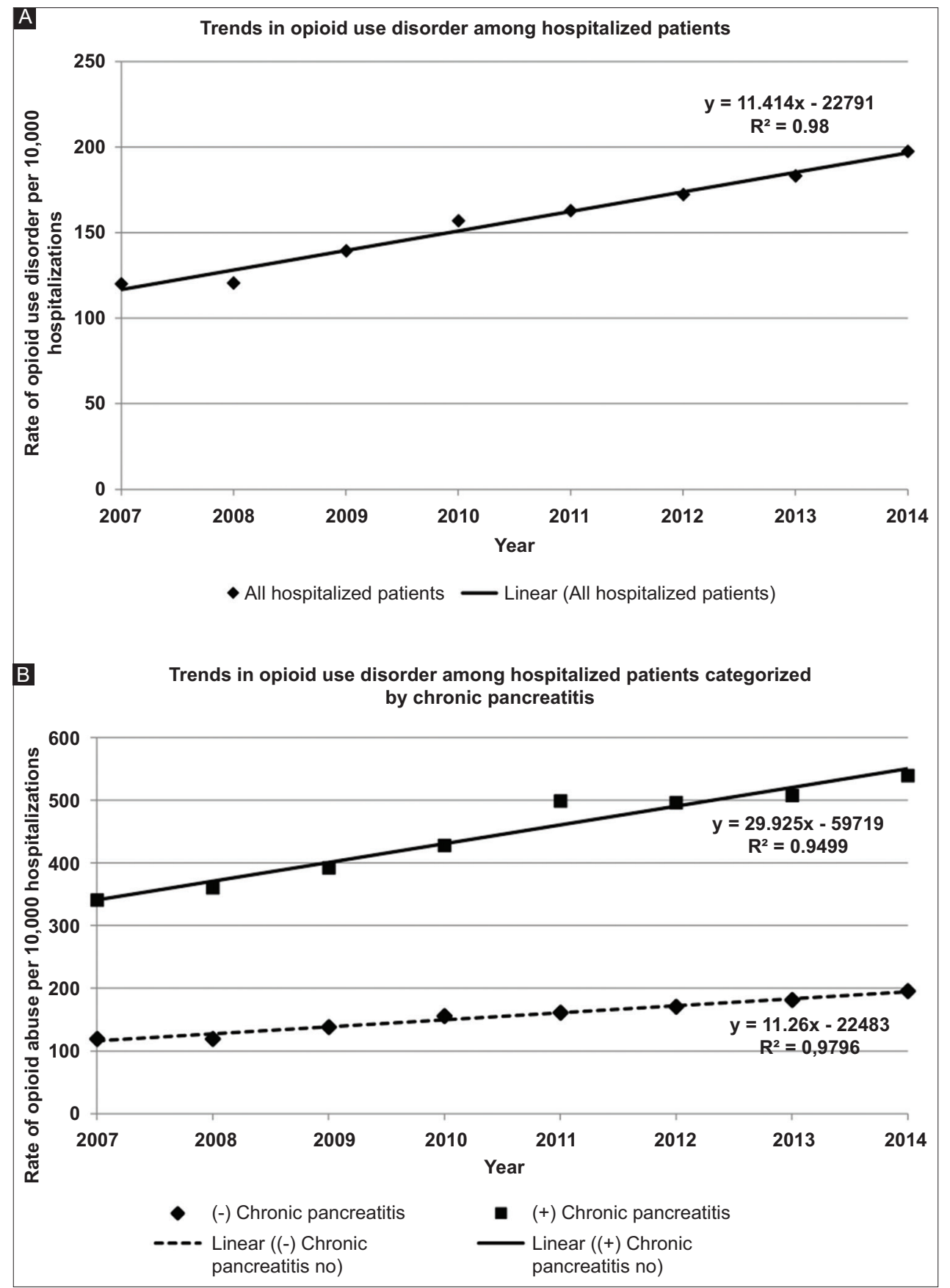

Figure 2 Annual prevalence of opioid use disorder among hospitalizations in the United States, 2007-2014. (A) Trends among all hospitalizations. (B) Trends among hospitalizations with vs. without chronic pancreatitis

supplementation and risk factor modification, including alcohol and tobacco abstinence [18,20,48,49]. An early surgical approach to treatment in qualified individuals has also been shown to be associated with improved outcomes [21,50-52]. Should opioids be required, patients should be educated and counseled on the potential side effects, and especially the risks of dependence and addiction [14]. As far as possible, opioids should serve as a bridge to a more sustainable treatment. They should be utilized at the minimum effective dose and duration [53]. Early referral of high-risk patients to a pain specialist, anesthesiologist, endoscopist or surgeon, as indicated, may be prudent [51].

There was a significant association between mental disorders, Medicare and Medicaid insurance, and opioid abuse in patients hospitalized with CP. Depressive symptoms were reported to be associated with opioid misuse among patients with CP in the single-center study by Barth et al [23]. Psychiatric disorders have consistently been shown to predict OUD in multiple studies of patients with IBD, which is another potent gastrointestinal cause of chronic relapsing/remitting 
abdominal pain $[42,54,55]$. This study also reveals a higher risk of OUD among patients insured with Medicaid and Medicare. With the expansion of Medicaid to cover healthcare for patients with substance abuse [56], CP patients with OUD are more likely to be Medicaid-insured. In a recent national study by Jeffery et al, opioid use rate was also found to be highest in Medicare vs. commercially-insured patients [57]. Likewise, chronic pain conditions, such as arthritis and back pain, were also identified as risk factors for opioid abuse, consistently with studies on other subsets of patients $[58,59]$. As in previous studies of acute pancreatitis [60] or other disease conditions, we found that females had higher odds of OUD. In addition to being more likely to be prescribed opioids at higher doses and longer duration for chronic pain [61,62], women are also biologically more susceptible to opioid dependence and addiction [63]. The higher odds of OUD among CP patients on government insurance (Medicare and Medicaid) compared to private insurance may be due to recent policy changes by private insurance companies to encourage better implementations of optimal prescription practice [64].

Patients with OUD had a longer LOS, which could be related to this patient group having a greater need for parenteral opiates and the longer time it may take to wean off these medications and transition to oral alternatives. Other plausible explanations include likely higher inpatient complications related to OUD, such as respiratory distress, constipation or altered mental status. Similarly, longer hospitalization stays have been described in IBD and orthopedic surgery patients with concomitant OUD [42,65]. While our analysis does not include data on the post-hospitalization outcomes of patients with CP with a diagnosis of opioid misuse, an understanding of the epidemiology of OUD in these patients is crucial to appreciating its potentially larger burden on the community. Since patients with CP show a higher OUD prevalence than both the population at large and other patient subgroups, and this prevalence is continuously rising, it is plausible that OUD in CP patients may be a major contributor to outpatient opioid-related complications and healthcare utilization. The similar in-hospital mortality rate and charges for CP patients with OUD and those without in our study only reflect events inside the hospital. Patient with OUD are known to have a higher prevalence of out-of-hospital opioid overdose, resulting in respiratory and cardiovascular arrest and, ultimately, in higher mortality $[66,67]$.

This study was limited by the use of a de-identified database without access to individual patients or charts; thus, we are only able to determine the predictors of opioid abuse among hospitalized patients with CP and not the specific underlying reasons for this behavior. Additional studies are needed to elucidate the specific causes of OUD in this patient group. As the unit of entry in the NIS database is a hospitalization and not a specific individual, there may be multiple hospitalizations for the same person. Furthermore, other associated factors that may elevate the risk of OUD, such as pain severity or CP duration, were not available. Similarly, clinical details, such as imaging features of the pancreas (degree of calcification, enlargement, pancreatic duct diameter) and frequency of past endoscopic/surgical pancreatic procedures, were not available.
Limited to only inpatient data, we could not determine the potential influence of inpatient care on post-hospitalization outcomes. Despite these limitations, our population-based study has the strengths of including a large sample size and being nationally illustrative of the escalating burden of OUD in patients with $\mathrm{CP}$, as well as its associated predictors.

To conclude, OUD affects a significant proportion of patients with $\mathrm{CP}$ and occurs at an increasing rate, almost 3 times the rate of those without CP. Young, female, Medicaidand Medicare-insured patients are most susceptible. Other risk factors are mental health disorders, pain-associated conditions and non-opioid drug abuse. A recognition of predisposing preexisting demographic and clinical variables may help clinicians to better identify individuals who are most at risk of opioid dependence and abuse, guiding a more prudent utilization of opioids and alternative therapy in this patient group, thereby slowing and potentially reversing the increase in rates of OUD.

\section{Summary Box}

\section{What is already known:}

- Patients with chronic pancreatitis (CP) suffer debilitating pain, which results in an elevated risk of opioid prescription and opioid use disorder (OUD)

- Research on the prevalence, risk factors and outcomes of OUD is lacking among patients with $\mathrm{CP}$

\section{What the new findings are:}

- The prevalence of OUD was 3 times higher among patients with $\mathrm{CP}$ vs. non-CP

- From 2007-2014, the prevalence of OUD increased among all hospitalizations, but at an almost 3 times higher rate among $\mathrm{CP}$ vs. non-CP patients

- Prevalent risk factors for OUD among CP patients include younger age, White vs. Hispanic race, hospitalizations in urban centers, and use of alcohol and other substances

- OUD was associated with a longer hospital stay, but no difference in mortality or hospital charges

\section{References}

1. Kanouse $\mathrm{AB}$, Compton $\mathrm{P}$. The epidemic of prescription opioid abuse, the subsequent rising prevalence of heroin use, and the federal response. J Pain Palliat Care Pharmacother 2015;29:102-114.

2. Dart RC, Surratt HL, Cicero TJ, et al. Trends in opioid analgesic abuse and mortality in the United States. $N$ Engl $J$ Med 2015;372:241-248.

3. Saha TD, Kerridge BT, Goldstein RB, et al. Nonmedical prescription opioid use and DSM-5 nonmedical prescription opioid use disorder in the United States. J Clin Psychiatry 2016;77:772-780. 
4. Manubay JM, Muchow C, Sullivan MA. Prescription drug abuse: epidemiology, regulatory issues, chronic pain management with narcotic analgesics. Prim Care 2011;38:71-90.

5. Module 5: Assessing and Addressing Opioid Use Disorder (OUD). Available from: https://www.cdc.gov/drugoverdose/training/oud/ accessible/index.html [Accessed 21 November 2020].

6. Chen LH, Hedegaard H, Warner M. Drug-poisoning deaths involving opioid analgesics: United States, 1999-2011. NCHS Data Brief 2014;(166):1-8.

7. Rudd RA, Aleshire N, Zibbell JE, Gladden RM. Increases in drug and opioid overdose deaths-United States, 2000-2014. MMWR Morb Mortal Wkly Rep 2016;64:1378-1382.

8. Kane-Gill SL, Rubin EC, Smithburger PL, Buckley MS, Dasta JF. The cost of opioid-related adverse drug events. J Pain Palliat Care Pharmacother 2014;28:282-293.

9. Herzig SJ, Rothberg MB, Cheung M, Ngo LH, Marcantonio ER. Opioid utilization and opioid-related adverse events in nonsurgical patients in US hospitals. J Hosp Med 2014;9:73-81.

10. Mudumbai SC, Lewis ET, Oliva EM, et al. Overdose risk associated with opioid use upon hospital discharge in veterans health administration surgical patients. Pain Med 2019;20:1020-1031.

11. Veiga DR, Mendonça L, Sampaio R, Lopes JC, Azevedo LF. Incidence and health related quality of life of opioid-induced constipation in chronic noncancer pain patients: a prospective multicentre cohort study. Pain Res Treat 2018;2018:5704627.

12. Mcdonald JP, Torrey AJ, Mehta MP, Delima LG. Beyond respiratory depression and constipation: adverse effects of opioids. J Miss State Med Assoc 2015;56:193-195.

13. Eriksen J, Sjøgren P, Bruera E, Ekholm O, Rasmussen NK. Critical issues on opioids in chronic non-cancer pain: an epidemiological study. Pain 2006;125:172-179.

14. CDC Guideline for Prescribing Opioids for Chronic Pain — United States, 2016. MMWR Recomm Rep 2016;65:1-49

15. Bao Y, Wen K, Johnson P, Jeng PJ, Meisel ZF, Schackman BR. Assessing the impact of state policies for prescription drug monitoring programs on high-risk opioid prescriptions. Health Aff (Millwood) 2018;37:1596-1604.

16. Conwell DL, Lee LS, Yadav D, et al. American Pancreatic Association Practice Guidelines in Chronic Pancreatitis: evidence-based report on diagnostic guidelines. Pancreas 2014;43:1143-1162.

17. Czul F, Coronel E, Donet JA. Update on chronic pancreatitis: review article. Rev Gastroenterol Peru 2017;37:146-155.

18. Johnstone CC. An overview of the management of patients with chronic pancreatitis. Nurs Stand 2016;31:54-63.

19. Khalid A, Whitcomb DC. Conservative treatment of chronic pancreatitis. Eur J Gastroenterol Hepatol 2002;14:943-949.

20. Enweluzo C, Tlhabano L. Pain management in chronic pancreatitis: taming the beast. Clin Exp Gastroenterol 2013;6:167-171.

21. Gupte AR, Forsmark CE. Chronic pancreatitis. Curr Opin Gastroenterol 2014;30:500-505.

22. Nusrat S, Yadav D, Bielefeldt K. Pain and opioid use in chronic pancreatitis. Pancreas 2012;41:264-270.

23. Barth KS, Balliet W, Pelic CM, et al. Screening for current opioid misuse and associated risk factors among patients with chronic nonalcoholic pancreatitis pain. Pain Med 2014;15:1359-1364.

24. HCUP-US NIS Overview. Available from: https://www.hcup-us. ahrq.gov/nisoverview.jsp\#data [Accessed 21 November 2020].

25. Rothstein MA. Is deidentification sufficient to protect health privacy in research? Am J Bioeth 2010;10:3-11.

26. 45 CFR 46. Available from: https://www.hhs.gov/ohrp/regulationsand-policy/regulations/45-cfr-46/index.html [Accessed 21 November 2020].

27. Adejumo AC, Adejumo KL, Pani LN. Risk and outcomes of Clostridium difficile infection with chronic pancreatitis. Pancreas 2019;48:1041-1049.
28. Adejumo AC, Akanbi O, Adejumo KL, Bukong TN. Reduced risk of alcohol-induced pancreatitis with cannabis use. Alcohol Clin Exp Res 2019;43:277-286.

29. Akanbi O, Adejumo AC, Soliman M, Kudaravalli P. Chronic pancreatitis patients who leave against medical advice: prevalence, trend, and predictors. Dig Dis Sci 2020 May 2 [Online ahead of print]. doi: 10.1007/s10620-020-06279-2

30. Healthcare Cost and Utilization Project (HCUP) NIS notes. Available from: https://www.hcup-us.ahrq.gov/db/vars/hosp_ bedsize/nisnote.jsp [Accessed 21 November 2020].

31. Healthcare Cost and Utilization Project (HCUP) NIS Notes. Available from: https://www.hcup-us.ahrq.gov/db/vars/h_contrl/ nisnote.jsp [Accessed 21 November 2020].

32. Quan H, Sundararajan V, Halfon P, et al. Coding algorithms for defining comorbidities in ICD-9-CM and ICD-10 administrative data. Med Care 2005;43:1130-1139.

33. Akanbi O, Adejumo AC, Saleem N, Francisque F, Soliman M, Ogunbayo GO. Sickle cell disease is associated with higher mortality among patients hospitalized with ischemic bowel disease. Eur J Gastroenterol Hepatol 2018;30:1027-1032.

34. Adejumo AC, Adejumo KL, Adegbala OM, et al. Protein-energy malnutrition and outcomes of hospitalizations for heart failure in the USA. Am J Cardiol 2019;123:929-935.

35. Adejumo AC, Ajayi TO, Adegbala OM, et al. Cannabis use is associated with reduced prevalence of progressive stages of alcoholic liver disease. Liver Int 2018;38:1475-1486.

36. Onyeakusi NE, Mukhtar F, Gbadamosi SO, et al. Cancer-related pain is an independent predictor of in-hospital opioid overdose: a propensity-matched analysis. Pain Med 2019;20:2552-2561.

37. Hale J, Thompson D, Darden P. Calculating subset weighted analysis using PROC SURVEYFREQ and GENMOD. Available from: http:// support.sas.com/resources/papers/proceedings13/272-2013.pdf [Accessed 21 November 2020].

38. HCUP Methods Series Calculating National Inpatient Sample (NIS) Variances for Data Years 2012 and Later. Available from: https://www.hcup-us.ahrq.gov/reports/methods/2015_09. jsp\#appa [Accessed 21 November 2020].

39. Singh VK, Drewes AM. Medical management of pain in chronic pancreatitis. Dig Dis Sci 2017;62:1721-1728.

40. Alexander LM, Keahey D, Dixon K. Opioid use disorder: a public health emergency. JAAPA 2018;31:47-52.

41. Han B, Compton WM, Blanco C, Crane E, Lee J, Jones CM. Prescription opioid use, misuse, and use disorders in U.S. adults: 2015 national survey on drug use and health. Ann Intern Med 2017;167:293-301.

42. Cohen-Mekelburg S, Rosenblatt R, Gold S, et al. The impact of opioid epidemic trends on hospitalised inflammatory bowel disease patients. J Crohns Colitis 2018;12:1030-1035.

43. Dewan KC, Dewan KS, Idrees JJ, et al. Trends and outcomes of cardiovascular surgery in patients with opioid use disorders. JAMA Surg 2019;154:232-240.

44. Yang S, Werner BC. Risk factors for prolonged postoperative opioid use after spinal fusion for adolescent idiopathic scoliosis. J Pediatr Orthop 2019;39:500-504.

45. Edlund MJ, Steffick D, Hudson T, Harris KM, Sullivan M. Risk factors for clinically recognized opioid abuse and dependence among veterans using opioids for chronic non-cancer pain. Pain 2007;129:355-362.

46. Bedard NA, DeMik DE, Dowdle SB, Callaghan JJ. Trends and risk factors for prolonged opioid use after unicompartmental knee arthroplasty. Bone Joint J 2018;100-B(1 Supple A):62-67.

47. Ahmed A, Yakah W, Freedman SD, Kothari DJ, Sheth SG. Evaluation of opioid use in acute pancreatitis in absence of chronic pancreatitis: absence of opioid dependence an important feature. Am J Med 2020;133:1209-1218. 
48. Olesen SS, Juel J, Graversen C, Kolesnikov Y, Wilder-Smith OH, Drewes AM. Pharmacological pain management in chronic pancreatitis. World J Gastroenterol 2013;19:7292-7301.

49. Sachdev AH, Gress FG. Celiac plexus block and neurolysis: a review. Gastrointest Endosc Clin N Am 2018;28:579-586.

50. Yang D, Forsmark CE. Chronic pancreatitis. Curr Opin Gastroenterol 2017;33:396-403.

51. Skube ME, Beilman GJ. Surgical treatment of pain in chronic pancreatitis. Curr Opin Gastroenterol 2018;34:317-321.

52. D’Haese JG, Ceyhan GO, Demir IE, Tieftrunk E, Friess H. Treatment options in painful chronic pancreatitis: a systematic review. HPB (Oxford) 2014;16:512-521.

53. Geppetti P, Benemei S. Pain treatment with opioids : achieving the minimal effective and the minimal interacting dose. Clin Drug Investig 2009;29 Suppl 1:3-16.

54. Crocker JA, Yu H, Conaway M, Tuskey AG, Behm BW. Narcotic use and misuse in Crohn's disease. Inflamm Bowel Dis 2014;20:2234-2238.

55. Edwards JT, Radford-Smith GL, Florin TH. Chronic narcotic use in inflammatory bowel disease patients: prevalence and clinical characteristics. J Gastroenterol Hepatol 2001;16:1235-1238.

56. Substance Abuse and Mental Health Services Administration (US); Office of the Surgeon General (US). Facing Addiction in America: The Surgeon General's Report on Alcohol, Drugs, and Health [Internet]. Washington (DC): US Department of Health and Human Services; 2016 Nov. CHAPTER 6, HEALTH CARE SYSTEMS AND SUBSTANCE USE DISORDERS. Available from: https://www.ncbi.nlm.nih.gov/books/NBK424848/ [Accessed 21 November 2020].

57. Jeffery MM, Hooten WM, Henk HJ, et al. Trends in opioid use in commercially insured and Medicare Advantage populations in 2007-16: retrospective cohort study. BMJ 2018;362:k2833.
58. Pauly NJ, Michailidis L, Kindred MG, et al. Predictors of chronic opioid use in newly diagnosed Crohn's disease. Inflamm Bowel Dis 2017;23:1004-1010.

59. Hanson KA, Loftus EV Jr, Harmsen WS, Diehl NN, Zinsmeister AR, Sandborn WJ. Clinical features and outcome of patients with inflammatory bowel disease who use narcotics: a case-control study. Inflamm Bowel Dis 2009;15:772-777.

60. Wu BU, Butler RK, Chen W. Factors associated with opioid use in patients hospitalized for acute pancreatitis. JAMA Netw Open 2019 05;2:e191827.

61. Serdarevic M, Striley CW, Cottler LB. Sex differences in prescription opioid use. Curr Opin Psychiatry 2017;30:238-246.

62. Opioids and women: from prescription to addiction. NWHN 2018. Available from: https://www.nwhn.org/prescription-addictionopioid-epidemic/ [Accessed 21 November 2020].

63. Becker JB, McClellan ML, Reed BG. Sex differences, gender and addiction. J Neurosci Res 2017;95:136-147.

64. García MC, Dodek AB, Kowalski T, et al. Declines in opioid prescribing after a private insurer policy change - Massachusetts, 2011-2015. MMWR Morb Mortal Wkly Rep 2016;65:1125-1131.

65. Menendez ME, Ring D, Bateman BT. Preoperative opioid misuse is associated with increased morbidity and mortality after elective orthopaedic surgery. Clin Orthop Relat Res 2015;473:2402-2412.

66. Alqahtani S, Nehme Z, Williams B, Smith K. The incidence and outcomes of out-of-hospital cardiac arrest precipitated by drug overdose: A systematic review and meta-analysis. Resuscitation 2019;134:10-18.

67. Bohnert AS, Valenstein M, Bair MJ, et al. Association between opioid prescribing patterns and opioid overdose-related deaths. JAMA 2011;305:1315-1321. 


\section{Supplementary Table}

Supplementary Table 1 ICD-9-CM codes for identifying clinical conditions in the HCUP-NIS Dataset, 2007-2014

Clinical condition

ICD-9-CM codes, elixhauser comorbidity variables and clinical classification system codes

\begin{tabular}{lc}
\hline Chronic pancreatitis & 577.1 \\
\hline Opioid use disorder & $304.0,304.7,305.5$ \\
\hline Mental health disorders & $293,294,295,296,297,298,299,300,301,302$ \\
\hline Back pain & $720,721,722,723,724$ \\
\hline Arthritis & $710-719,725,726,727,728,729,730-739$ \\
\hline Headaches & $346,307.81,784.0$ \\
\hline Alcohol use & $303 . x, 305.3 x, 291 . x, 980 . x$, V11.3 \\
\hline Biliary stones & $574 . x$ \\
\hline Family history of pancreatitis & V18.59 \\
\hline Autoimmune diseases & $710 . x$ \\
\hline Pancreatic cancer & $156.2,157 . x$, V10.09 \\
\hline Hyercalcemia & 275.42 \\
\hline Hypertriglyceridemia & $272.1,272.2$ \\
\hline Cystic fibrosis & 277.0 \\
\hline Tobacco use & Quan H, Sundararajan V, Halfon P, et al. Coding algorithms for defining \\
\hline Other substance use (neither alcohol, tobacco nor opioid) & $305.1,989.84$, V15.82 \\
\hline Charlson-Deyo comorbidity index & $2005 ; 43: 1130-1139$. \\
\hline
\end{tabular}

NIS, National Inpatient Database (NIS); HCUP, Healthcare Cost and Utilization Project

Supplementary Table 2 Trends in opioid use disorder (cases per 10,000 hospitalizations) among adult hospitalizations, categorized by presence of chronic pancreatitis, NIS 2007-2014

\begin{tabular}{lccc}
\hline \multirow{2}{*}{ Year } & \multirow{2}{*}{ All patients } & \multicolumn{2}{c}{ Chronic pancreatitis } \\
\cline { 3 - 4 } & & No & Yes \\
\hline 2007 & 135.0 & 134.2 & 365.3 \\
2008 & 137.1 & 136.1 & 386.7 \\
\hline 2009 & 156.1 & 154.9 & 421.5 \\
2010 & 175.7 & 174.3 & 453.2 \\
2011 & 182.1 & 180.4 & 527.5 \\
2012 & 192.0 & 190.3 & 521.4 \\
\hline 2013 & 202.4 & 200.6 & 534.6 \\
2014 & 216.4 & 214.5 & 566.2 \\
\hline $2007-2014$ & 174.0 & 172.5 & 478.8 \\
\hline
\end{tabular}

NIS, National Inpatient Database 